\title{
Do learning communities encourage potential STEM majors?
}

\author{
Alexis V. Knaub, ${ }^{1}$ Jenifer N. Saldanha, ${ }^{2}$ Clark R. Coffman, ${ }^{2}$ and Charles R. Henderson ${ }^{3}$ \\ ${ }^{1}$ Center for Research on Instructional Change in Postsecondary Education (CRICPE), Western Michigan University, Kalamazoo, MI, 49008 \\ ${ }^{2}$ Department of Genetics, Development, and Cell Biology, Iowa State University, Ames, IA 50011 \\ ${ }^{3}$ Department of Physics, Western Michigan University, Kalamazoo, MI, 49008
}

\begin{abstract}
Some students enter college with undeclared majors but could be STEM majors with encouragement. One way of encouraging these students is to provide these students with rich, interactive experiences through learning communities dedicated to students with undeclared STEM majors. Although learning communities are designed to be interactive, it is unclear whether they are and if interactions are linked to any outcomes for students. We used social network analysis (SNA) to study first-year student learning community networks for discussion, problem solving, and social reasons. Using chi-square tests, we also examined whether learning community network behaviors are linked to any outcomes. Results show that students have ties. However, in-degree centrality, a measure of how many respondents select an individual, does not have any relationship with the examined outcomes.
\end{abstract}

\section{INTRODUCTION}

In the US, the need for STEM workers outpaces the number of STEM degree recipients [1]. Students entering college without declared majors could become STEM majors if encouraged. One recommended way to encourage pursuing STEM is through student learning communities [2]. Student learning communities are designed to foster peer interaction and collaborative learning [3]. At Iowa State University (ISU), students with undeclared majors participated in a learning community consisting of five teams that focused on highlighting STEM careers and "big picture" areas such as environmental conservation and big data. While research on student learning communities suggest positive results such as persistence, we do not know whether they can motivate undeclared students to declare STEM majors.

Social interaction is central in a learning community but may not frequently occur in practice. It is unclear whether students have ties and with whom as well as in which contexts. It is also unclear whether the social aspects have any impact on outcomes and if so, how early they can be seen. We use social network analysis (SNA), a tool that can be used to quantitatively study social interactions, and other survey data to present preliminary results on this learning community. Our research questions are: do learning community students have ties within their teams? Are any outcomes are related to these ties?

\section{BACKGROUND}

\section{A. What are student learning communities?}

Student learning communities are collaborative environments where students participate in activities that allow them to socially construct knowledge and develop critical thinking skills $[3,4]$. The content in learning communities is multidisciplinary in order for students to gain a deeper understanding [3]. For example, a learning community might focus on healthcare careers and show how biology, chemistry, and physics are relevant to such careers.

Student learning communities are linked to positive outcomes. Learning community students often spend more time working together and find their social and academic lives intertwined [3]. This supports their persistence. They become integrated into the institution, engaged in educational activities, and generally have positive experiences with the institution [4]. Effects are found more strongly in first-year students but can be seen even in senior students [4]. In STEM, learning communities are linked to science identity development and persistence [2]. These learning communities may also contribute to students' science capital, which combines social capital (e.g., knowing and interacting with scientists) and cultural capital (e.g., scientific literacy, understanding scientific practices) [5]. Having more science capital is associated with science identity (i.e., others identify an individual as a science person) [5]. Being acknowledged by others as a science person contributes to students feeling that they belong in a field [2], which may support persistence in STEM.

\section{B. What is SNA, and why use it for learning communities?}

Social network analysis (SNA) is a means to study ties to other actors, making it suitable to study group interactions. In education, SNA been used to study online learning communities [6] and learning communities associated with a department in a traditional sequence course [7], but has not been used to study learning communities aimed at encouraging the declaration of STEM majors.

We primarily examined in-degree network properties, which consider how many respondents indicated a tie to an actor. In-degree is of interest because frequently selected actors are considered popular for a purpose $[8,9]$. In the context of this paper, being frequently selected suggests that respondents see the actor as someone who is valued in science. Being frequently selected may contribute to science identity. Although actors do not know whether they were selected, we infer that actors are aware of whether they are included be- 
cause ties are associated with activities.

The value of social networks is not simply that one knows or is known by many. Often whom one knows is important. This is one form of social capital, where ties to particular actors may allow one to reap benefits [9]. We also examined out-degree, to whom respondents indicated a tie, with the focus on their learning community leaders or peer mentors. We hypothesized having a tie to an authoritative figure would be positively related to desired outcomes.

\section{METHODOLOGY}

\section{A. Setting}

ISU is a public research-intensive institution that has engaged for the past 7 years in multiple projects funded by the Howard Hughes Medical Institute (HHMI) to improve undergraduate STEM education and increase the number of STEM undergraduates completing a degree. The learning community in this study supports these goals. Students with undeclared majors can enroll in learning community teams designed to encourage declaring a STEM major. Activities provide exploration of majors and careers. Faculty and staff serve as facilitators and are referred to as team leaders. Peer mentors are student facilitators in their sophomore year or higher. These teams are run during the fall semester, but students can pursue research during the spring semester.

\section{B. Data collection and analysis}

At the end of fall 2016, all first-year students in one learning community consisting of 5 teams $(N=85)$ dedicated to undeclared potential STEM majors were invited to participate in a survey with the following SNA questions:

1. Whom do you meet for learning community related reasons? For example: meeting to discuss challenges within the learning community, team work/project/activities, and similar activities.

2. Whom do you meet for social reasons? For example: having a meal, watching TV, seeking personal advice, and similar activities.

3. Whom do you meet for problem solving/concept discussion? For example: working on schoolwork.

Questions \#1 and 3 were asked because they are related to academic activitities that may contribute science identity. Question \#2 looks at the purely social aspect of networks because socializing is linked to persistence [3].

Students selected up to 10 actors from a roster of members in their team. The roster included fellow first-year students, team leaders, and peer mentors. They also could provide 10 additional names. The survey contained questions regarding experiences with both the learning community (e.g., did they receive satisfactory course support?) and the general institution (e.g., have they visited a faculty member or their advisor?). This survey was piloted with the peer mentors and modifications were made based on their comments.

Data were analyzed using UCINET [10] and SPSS 23. These results include 3 of the 5 learning community teams due to low response rates for two teams. The only identifiable difference for those teams is that the first-year students infrequently responded to emails for the course.

Teams had between 14 and 22 first-year students. Team A had a response rate of $57 \%(N=12)$, B's was $68 \%(N=$ $15)$, and C's was 57\% $(N=8)$. All respondents completed the survey. In calculating A's response rate, we omitted 3 students who did not receive the survey due to a clerical error. They are otherwise included in these data whenever they were selected by respondents.

\section{Limitations}

One limitation to this study is that this is a case study at one institution. These results may change at a different institution with different constraints and affordances. However, ISU shares many commonalities with other institutions (e.g., there are many large research institutions) and similar institutions are engaged in similar work.

Another limitation is that the networks are incomplete as we do not have a $100 \%$ response rate. Had the nonrespondents participated in the study, results might slightly change. Although non-respondents are a common issue with survey-based SNA data, there is no standard method to handle them, with most options being quite exploratory (e.g., exponential random graph modeling) and introducing other issues as they make assumptions about non-respondents' ties [11]. For the teams that are included, the response rates range from $57-68 \%$. In-degree tends to be fairly stable when $50 \%$ or higher of all responses is available [12].

Lastly, these are preliminary results taken after one semester. Learning community effects may need more time to appear. However, preliminary results can be useful to know whether the learning community functions as intended and if there are early signs of success, such as interest in STEM.

\section{PRELIMINARY RESULTS}

\section{A. Student learning community networks}

We examined in-degree centrality to see if students have few or many ties. In-degree centrality is calculated by adding the total number of respondents who selected an actor. Unless otherwise specified, centrality refers to in-degree centrality. Table I displays the average centrality and standard error for first-year students. $N$ is the number of students in each team and includes both respondents and non-respondents. Raw 
centrality scores were normalized to the number of possible nominations. The closer the average is to 1 , the more respondents selected that actor; if the average were 1, all respondents selected the actor. For all three learning community sections, centrality for problem-solving and social reasons is lower than that of discussion. Except for social reasons, team C's students tend to have the highest centrality. This means that team C's students tend to be identified more often as ties for discussion (26\% of the time) and problem-solving (4\%).

TABLE I. In-degree centrality of learning community students

\begin{tabular}{ccccc}
\hline \hline & $N$ & Discussion & Problem-solving & Social \\
\hline Team A & 21 & $0.15 \pm 0.02$ & $0.08 \pm 0.07$ & $0.01 \pm 0.01$ \\
Team B & 22 & $0.14 \pm 0.02$ & $0.04 \pm 0.02$ & $0.09 \pm 0.01$ \\
Team C & 14 & $0.26 \pm 0.04$ & $0.11 \pm 0.03$ & $0.05 \pm 0.02$ \\
\hline \hline
\end{tabular}

We used ANOVAs to determine whether there are any statistical differences among the groups. There is a statistically significant difference $(p<0.05)$ for the discussion network, $F(2,57)=5.72$. A post-hoc Tukey test indicates the difference between team $\mathrm{C}$ and team $\mathrm{A}$, as well as team $\mathrm{C}$ and team $\mathrm{B}$, are statistically significant $(p<0.05)$. We note that team $\mathrm{C}$ is also the smallest of the three sections, which may lead to higher centrality for discussion and for problem-solving; they had fewer options for ties. When asked, the leaders did not observe any major differences in how each team operated.

We examined centrality for peer mentors and team leaders. These results are in Table II. Centrality for both peer mentors and leaders tend to be higher than the average for peers, meaning that respondents tended to select peer mentors and leaders more often than peers. This may be because there are fewer people in these roles and thus, fewer options. Peer mentors tend to be selected more than team leaders. Team leaders noted that they had discussions with the peer mentors regarding the importance of engaging the students which may have influenced peers mentors to engage to students.

TABLE II. In-degree centrality of learning community peer mentors and team leaders

\begin{tabular}{ccccc}
\hline \hline & $N$ & Discussion & Problem-solving & Social \\
\hline Peer mentor & 8 & $0.38 \pm 0.08$ & $0.12 \pm 0.03$ & $0.13 \pm 0.02$ \\
Team leaders & 4 & $0.25 \pm 0.10$ & $0.11 \pm 0.05$ & $0.02 \pm 0.02$ \\
\hline \hline
\end{tabular}

To determine whether ties are evenly distributed among actors (students, peer mentors, and team leaders) in the learning community, we examined in-degree centralization. Centralization characterizes the overall network and measures whether actors have a similar number of ties or a few actors have the majority of ties (e.g., some actors are more "popular" than others) [8]. In our case, we were interested in whether actors in the network have similar number of ties. Centralization is on a 0 to 1 scale, where 0 is when all actors have an equal number of ties and 1 is when a single actor has the maximum centrality and others have no ties. Centralization, as defined by Freeman [8], is calculated as follows:

$$
C_{z}=\frac{\sum_{n=1}^{N} C_{M a x}-C_{n}}{(N-1)(N-2)}
$$

where $N$ is the number of actors in the network (respondents, non-respondents, team leaders, and peer mentors), $C_{M a x}$ is the maximum number of ties held by actor, and $C_{n}$ is the number of ties an actor has.

These results are displayed in table III. The percentage of actors with zero ties includes students, peer mentors, and team leaders. Team $\mathrm{C}$ has higher centralizations for all three purposes compared to teams $\mathrm{A}$ and $\mathrm{B}$. This means that some actors in team $\mathrm{C}$ were selected as ties more often than others, while actors in A or B were selected by a similar number of times. Team $\mathrm{C}$ also has the highest percentage of actors who have no centrality for social and discussion purposes, while team B has the highest percentage for problem-solving. In terms of centralization, it is not merely that some actors are more popular than others. Some actors have many ties and others are not acknowledged by respondents as part of the network.

\section{B. Outcomes associated with networks}

We used chi-square tests to determine whether ties had any relationship with: whether students have visited their advisor/non-learning community faculty member and whether students feel they receive satisfactory course material support. The tie variables examined were: being selected at least once by a peer, having at least one peer mentor tie, or having at least one team leader tie. The latter two tie variables are out-degree, i.e., the variable is whether respondents indicated a tie.

The tie variables were selected because we hypothesized that it is not the number of ties but simply having a tie that matters. Peer ties may be important for inclusion, peer mentor ties for role models, and team leader ties for access to a scientist. The first outcome was selected because we hypothesized that being part of the network would encourage students to visit non-learning community faculty. The second outcome was selected because we hypothesized that having ties might lead to students seeking out course support, e.g., being encouraged by others to attend study groups.

Twenty-three out of 35 respondents $(66 \%)$ had contacted an advisor or a non-learning community faculty member. Twenty-four $(69 \%)$ reported receiving satisfactory course support. There is no statistically significant $(p<0.05)$ interaction with any of the tie variables and the outcome variables. Our data do not support our hypotheses.

Data collection took place when students were in their first semester of their first year. We currently cannot determine whether centrality is linked to declaring a STEM major. However, we know that five students decided to pursue STEM research in the second semester. Teams B and C had students 
TABLE III. In-degree centralization. Network actors include learning community students, peer mentors, and team leaders

\begin{tabular}{cccccccc}
\hline \hline & $N$ & Discussion & $\begin{array}{c}\% \text { with 0 ties } \\
\text { for discussion }\end{array}$ & Problem-solving & $\begin{array}{c}\% \text { with 0 ties } \\
\text { for problem-solving }\end{array}$ & $\begin{array}{c}\text { Social } \\
\% \text { with ties } \\
\text { for social }\end{array}$ \\
\hline Team A & 29 & 0.08 & $0 \%$ & 0.07 & $28 \%$ & 0.08 & $31 \%$ \\
Team B & 26 & 0.08 & $8 \%$ & 0.11 & $65 \%$ & 0.08 & $23 \%$ \\
Team C & 17 & 0.19 & $12 \%$ & 0.15 & $35 \%$ & 0.11 & $65 \%$ \\
\hline \hline
\end{tabular}

who did research. On average, students who pursued research tended to have lower centrality for problem-solving $(0.03 \pm$ $0.03)$ and social reasons $(0.03 \pm 0.02)$ than the students who did not pursue research $(0.07 \pm 0.01$ and $0.07 \pm 0.01$, respectively). Students who pursued did have slightly higher discussion centrality $(0.19 \pm 0.07)$ than students who did not $(0.16 \pm 0.01)$, meaning others considered them as discussion partners. We state these tentatively, as few students pursued research, and thus, we cannot test the outcome variable (second semester STEM research).

\section{DISCUSSION AND PRELIMINARY CONCLUSIONS}

The students in these three learning community teams have formed networks for learning community discussion, problem-solving, and social reasons. Although team leaders and peer mentors receive the same training and are working towards the same purpose, team $\mathrm{C}$ is different from the other two. Team $\mathrm{C}$ has a less equal distribution of ties among the actors in all three networks meaning that some actors in team 3 are selected more often than others. On average, students in team $\mathrm{C}$ have higher centrality than those in A or B. This perhaps is a function of size. The only noticeable difference between team $\mathrm{C}$ and teams $\mathrm{A}$ and $\mathrm{B}$ is that team $\mathrm{C}$ has fewer students (14) than A (21) or B (22).

Students formed networks with their peer mentors and team leaders. Both peer mentors and team leaders have higher centrality than that in the peer networks. This may be a result of students seeking out those in charge of the learning communities and having fewer options if one seeks out someone in a more authoritative role than they would with a peer. Team leaders and peer mentors are also encouraged to connect with the students, which may also help foster these ties.

We also examined these SNA data with outcome data: whether students visited a non-learning community faculty member or their advisor, whether they feel they have had satisfactory support for course materials, and whether they pursued optional STEM research in second semester. We hypothesized that being selected at least once by a peer, selecting a peer mentor as a tie, or selecting team leader as a tie would be related to these outcomes as both are associated with science identity. We found no relationship between the first two variables and SNA data. Due to few students continuing with research, our results are tentative. Students who continued with research have slightly higher centrality for learning community discussion, but they have lower centrality for social and problem-solving reasons. Having higher centrality for discussion may support their science identity development and thus, encourage them to pursue STEM research. However, it is perplexing why they would not have higher in-degree centrality for problem-solving.

Although our results mostly support null hypotheses, that in-degree centrality is not related to any outcome, these are results after only one semester. It may take more time to see any differences. We plan on comparing these learning community students to the control group, students with undeclared major who were in a different course that was not designed to encourage students to declare STEM majors. There are multiple future outcomes to examine including whether: these networks continue beyond their learning community semester; these students engage in other scientific activities such as summer research; and these students in this learning community declare STEM majors.
[1] The National Academies. Rising Above the Gathering Storm. (National Academies Press, Washington, DC, 2007).

[2] M. J. Graham, J. Frederick, A. Byars-Winston, A. B. Hunter, and J. Handelsman. Science. 341, 1455 (2013).

[3] V. Tinto. Higher Education Monograph Series. 1, 1 (2003).

[4] C. M. Zhao and G. D. Kuh. Research in Higher Education. 45, 115 (2004).

[5] L. Archer, E. Dawson, J. DeWitt, A. Seakins, and B. Wong. Journal of Research in Science Teaching. 52, 922 (2015).

[6] H. Cho, G. Gay, B. Davidson, and A. Ingraffea. Computers \& Education. 49, 309 (2007).

[7] E. Brewe, L. Kramer, and V. Sawtelle. Phys. Rev. Phys. Educ.
Res. 8, 010101 (2012).

[8] S. Wasserman and K. Faust. Social Network Analysis: Methods and Applications. (Cambridge University Press, Cambridge, 1994).

[9] C. Kadushin. Understanding Social Networks. (Oxford University Press, Oxford, 2012).

[10] S.P. Borgatti, M.G. Everett, and L.C. Freeman, software Ucinet for Windows, Analytic Technologies, Harvard, MA, 2002.

[11] J.F. Morris and R.F. Deckro. Behavioral Sciences of Terrorism and Political Aggression. 5, 70 (2013).

[12] E. Costenbader and T.W. Valente. Social Networks, 25, 283 (2003). 